\title{
Nanomagnetic Materials in Medicine: Recent Developments in Imaging, Diagnostics and Therapy.
}

Kannan M. Krishnan.

Department of Materials Sciences \& Engineering, University of Washington, Seattle, WA, USA

The Néel relaxation of magnetic nanoparticles (MNP), subject to alternating magnetic fields in solution, depends exponentially on the size of their magnetic core, whilst the complementary Brownian relaxation mechanism depends critically on their hydrodynamic volume [1,2]. Recent developments in the synthesis [3] and applications [4] of highly monodisperse and phase-pure magnetite nanoparticles, with negligible toxicity [5], and favorable biodistribution [6], allows for reproducible control of the former, even in "harsh" biological environments, enabling novel imaging [7-9], spectroscopic [10] and therapeutic [11,12] modalities, under $a c$ excitations. With appropriate functionalization of the monodisperse cores, and utilizing the magnetic relaxation, it also allows for the development of sensitive, relaxation based assays [13] for the detection of biomolecules associated with various diseases.

In this brief talk, beginning with the physics of magnetic relaxation under ac excitation, I will outline the chemical synthesis, phase optimization, surface functionalization, and development of optimized nanoparticle tracers with magnetic properties tailored for the unique physics of Magnetic Particle Imaging - an emerging tracer-based whole body imaging technique with no ionizing radiation, and present recent results in phantom and in vivo imaging of animal models for cardiovascular disease and molecular imaging of cancer. I will also present a brief introduction to magnetic fluid hyperthermia as a therapeutic modality and the framework of a newly designed assay, using magnetic particle spectroscopy, for the detection of proteases that are closely linked to malignant phenotypes of different solid tumors.

I will conclude with an outlook of opportunities that abound for continued research in this exciting field [14].

References:

[1] Kannan M. Krishnan, IEEE Trans. Mag. 46 (2010), p. 2523.

[2] C. Kuhlmann et al, IEEE Trans. Mag. 51 (2015), p. 6500504.

[3] R. Hufschmid et al, Nanoscale, 7 (2015), p. 11142.

[4] Y. Bao et al, Jour Mater. Sci., 51 (2016), p. 513.

[5] H. Arami et al, Chem. Soc. Rev. 44 (2015), p. 8576.

[6] H. Arami et al, Biomaterials 52 (2015), p. 251.

[7] B. Gleich \& J. Weizenecker, Nature 435 (2005), p. 1214.

[8] R.M. Ferguson et al, IEEE Trans. Med. Imag. 34 (2015), p. 1077.

[9] A. Tomitaka et al, Nanoscale 7 (2015), p. 16890.

[10] S. A. Shah et al, Phys. Rev. B92 (2015), p. 094438.

[11] A. Khandhar et al, Jour. Biomedical Materials Research, Part A, 100A (2012), p. 728.

[12] S. Kalale, R. Narain and Kannan M. Krishnan, Jour. Mag. Mag. Mat. 321 (2009), p. 1377.

[13] S. Gandhi, H. Arami, Kannan M. Krishnan, Proc. Nat. Acad. Sci., (submitted) 
[14] This work was supported by US-NIH Grants 1RO1EB013689-01/NIBIB, 1R41EB013520-01, and 2R42EB013520-02A1. I would also like to thank all my students, post-doctoral fellows and collaborators, for their invaluable contribution to this work. I am also deeply indebted to the late Prof. Gareth Thomas, who not only got me started but has also been both a source of inspiration and support throughout my academic career. 